\title{
A19 ANTI-CCP ANTIBODIES ARE A COLLECTION OF ACPA THAT ARE CROSS-REACTIVE TO MULTIPLE CITRULLINATED ANTIGENS
}

A loan-Facsinay, ${ }^{1} \mathrm{H}$ el-Bannoudi, ${ }^{1} \mathrm{H}$ U Scherer, ${ }^{1}$ D van der Woude, ${ }^{1}$ H A Ménard, ${ }^{2}$ M Lora, ${ }^{2}$ L A Trouw, ${ }^{1} \mathrm{~T}$ W J Huizinga, ${ }^{1}$ R E M Toes ${ }^{1}$ Department of Rheumatology, Leiden University Medical Center, Leiden, The Netherlands; ${ }^{2}$ Division of Rheumatology, McGill University Health Center, Montreal, Quebec, Canada

10.1136/ard.2010.129577s

Objective Anti-citrullinated protein/peptide antibodies (ACPA/anti-CCP) are a hallmark of rheumatoid arthritis and are believed to play a role in disease pathogenesis. These antibodies are typically detected in ELISA with citrullinated peptides (eg, CCP2) or -proteins as antigens. The absolute concentration of anti-CCP antibodies in serum is unknown. Although antibodies to several citrullinated proteins can mainly be detected within anti-CCP-positive sera, it is currently unknown whether anti-CCP antibodies are in fact ACPA. Likewise, it is unknown to what extent antibody responses to different citrullinated proteins are cross-reactive.

Methods The authors established an affinity purification method in which citrullinated-antigen-specific antibodies were eluted from ELISA plates and then used for detection of citrullinated antigens in ELISA or Western Blot. For additional cross-reactivity studies, ELISA-based inhibition assays were performed with citrullinated or control peptides as inhibitors. Results The authors estimated the concentration of anti-CCP IgG antibodies to be at least $30 \mu \mathrm{g} / \mathrm{ml}$ in patients with high anti-CCP antibody levels. Affinity-purified anti-CCP antibodies were able to recognise citrullinated-fibrinogen (cit-fib) and cit-MBP on Western Blot. Furthermore, antibodies specific for cit-fib and cit-MBP were cross-reactive. However, additional cross-reactivity studies indicated that non-overlapping antibody responses to citrullinated peptides can exist in patients. Conclusions The authors show for the first time that antiCCP antibodies recognise multiple citrullinated proteins and are thus ACPA. More importantly, our data indicate that different ACPA responses are cross-reactive, but the cross-reactivity is not complete, as distinct non-cross-reactive responses can also be detected in RA patients. 